\title{
Measurement of Cosmic-Ray Nuclei with the Third Flight of the CREAM Balloon-Borne Experiment
}

\author{
J. R. Smith ${ }^{\dagger 1}$
}

E-mail: jacobuta@umd.edu

Y. Amare ${ }^{1}$, T. Anderson ${ }^{2}$, D. Angelaszek ${ }^{1,3}$, N. Anthony ${ }^{3}$, K. Cheryian ${ }^{3}$, G.H. Choi ${ }^{4}$, M. Copley ${ }^{1}$, S. Coutu' ${ }^{2}$, L. Derome ${ }^{5}$, L. Eraud ${ }^{5}$, L. Hagenau ${ }^{3}$, J.H. Han ${ }^{1}$, H.G. Huh ${ }^{1}$, S. Im $^{2}$, J.A. Jeon ${ }^{4}$, S. Jeong ${ }^{4}$, K.C. Kim ${ }^{1}$, M.H. Kim ${ }^{1}$, H.Y. Lee ${ }^{4}$, J. Lee ${ }^{4}$, M.H. Lee ${ }^{1}$, J. Liang $^{3}$, J.T. Link ${ }^{7}$, L. Lu ${ }^{3}$, L. Lutz ${ }^{1}$, A. Menchaca-Rocha ${ }^{7}$, T. Mernik ${ }^{1}$, J.W. Mitchell ${ }^{6}$, S.I. Mognet ${ }^{2}$, S. Morton ${ }^{3}$, M. Nester ${ }^{3}$, S. Nutter ${ }^{8}$, O. Ofoha ${ }^{1}$, I.H. Park ${ }^{4}$, N. PicotClemente $^{1}$, R. Quinn ${ }^{3}$, E.S. Seo ${ }^{1,3}$, P. Walpole ${ }^{1}$, R.P. Weinmann ${ }^{1}$, J.Wu ${ }^{1}$, Y.S. Yoon ${ }^{1}$

${ }^{1}$ Inst. for Phys. Sci. and Tech., University of Maryland, College Park, MD 20742, USA

${ }^{2}$ Dept. of Physics, Penn State University, University Park, PA 16802, USA

${ }^{3}$ Dept. of Physics, University of Maryland, College Park, MD 20742, USA

${ }^{4}$ Dept. of Physics, Sungkyunkwan University, Suwon 440-746, Republic of Korea

${ }^{5}$ Laboratoire de Physique Subatomique et de Cosmologie, UJF - CNRS/IN2P3 - INP, 53 rue des Martyrs, 38026 Grenoble Cedex, France

${ }^{6}$ Astroparticle Physics Laboratory, NASA Goddard Space Flight Center, Greenbelt, MD 20771, USA

${ }^{7}$ Instituto de Fisica, Universidad Nacional Autonoma de Mexico, Circuito de la Investigacion s/ $n$, Ciudad Universitaria, CP 04510 Mexico Distrito Federal, Mexico

${ }^{8}$ Dept. of Physics and Geology, Northern Kentucky University, Highland Heights, KY 41099, USA

The balloon-borne Cosmic Ray Energetics And Mass experiment had its third flight (CREAMIII) over Antarctica for 29 days from December 17, 2007 to January 19, 2008. CREAM-III was designed to directly measure the elemental spectra of cosmic-ray nuclei from Hydrogen to Iron in the energy range from $10^{12}$ to $10^{15} \mathrm{eV}$. Energy of incident cosmic rays was measured with a calorimeter that consisted of a densified carbon target directly above a stack of 20 alternating layers of tungsten and scintillating fiber ribbons. Multiple charge measurements were independently made with the silicon charge detector (SCD), Cherenkov Camera (CherCam), and a Timing Charge Detector (TCD) in order to identify particles and minimize backscattering effects from the calorimeter. Compared to previous CREAM flights, the electronic noise of CREAM-III was reduced, significantly lowering the energy threshold. Results from on-going analysis of the energy spectra will be presented.

35th International Cosmic Ray Conference - ICRC2017

10-20 July, 2017

Bexco, Busan, Korea

\section{${ }^{\dagger}$ Speaker}




\section{Introduction}

CREAM (Cosmic Ray Energetics And Mass) is a balloon experiment designed to perform direct measurements of cosmic-ray composition and energy spectra above the atmosphere. The goal of this multi-mission experiment is to collect sufficient statistics to explore the region of energies up to $10^{15} \mathrm{eV}$ and test the current models of cosmic-ray acceleration and propagation in the Galaxy. The third CREAM payload (CREAM-III) was launched from McMurdo on December 19, 2007 and landed on January 17 2008, for a 29 day flight. The balloon circumnavigated the Antarctic continent twice and floated at an altitude $\sim 38 \mathrm{~km}$ with an atmospheric overburden of $3.9 \pm 0.4 \mathrm{~g} / \mathrm{cm}^{2}$. One of the primary scientific goals of the CREAM project is the measurement of secondary cosmic-ray nuclei at high energies. Secondary nuclei are those which are produced predominantly through spallation interactions of primary nuclei, those produced in the cosmicray source regions. Because these spallation interactions are thought to occur primarily during the propagation of primary nuclei in the interstellar medium, a measurement of the ratio of secondary to primary fluxes reveals information about the propagation history (e.g., the amount of material traversed) of the primary particles In this paper, results from the on-going analysis of the data from the third flight are reported. The results presented in this paper are preliminary.

\section{CREAM-III Instrument and Flight}

Several detector systems make up the CREAM-III instrument: Timing Charge Detector, Cherekov Detector, Cherenkov Camera, Silicon Charge Detector, and Calorimeter below carbon targets as shown in Figure 1. The CAL provides a trigger and measures the energy of incident cosmic rays with a sampling calorimeter that consists of 20 alternating layers of tungsten (each 1 $\mathrm{X}_{0}$ ) and scintillating fiber ribbons (each $1 \mathrm{~cm}$ wide) and is mounted below a densified carbon target. The target has a nuclear interaction length of $0.5 \lambda_{\mathrm{L}}$ and the tungsten stack has a radiation length of $20 \mathrm{X}_{0}$ [1]. The SCD, CherCam and TCD measure the charge of incident cosmic rays. The SCD measures charge through energy loss, $\mathrm{dE} / \mathrm{dx}$, in the two independent silicon layers. The CherCam uses Cherenkov radiation generated by an aerogel layer and an array of PMTs [2], and the TCD uses energy loss through its plastic scintillators [3]. Each of the two SCD layers, top SCD (tSCD) and bottom SCD (bSCD) is segmented by an array of $2.12 \mathrm{~cm}^{2}$ pixels and provides two independent charge measurements with a resolution of $\sim 0.2 \mathrm{e}$ for protons and increases slightly up to Iron [4] . CREAM is the only multi-TeV cosmic-ray experiment to have all 3 charge measurement techniques to distinguish incident nuclei from back-scattered particles. For the analysis reported in this paper, the CAL is used to optimize event selection with energy-based trigger conditions and the two SCD layers provide charge measurement and identification. 


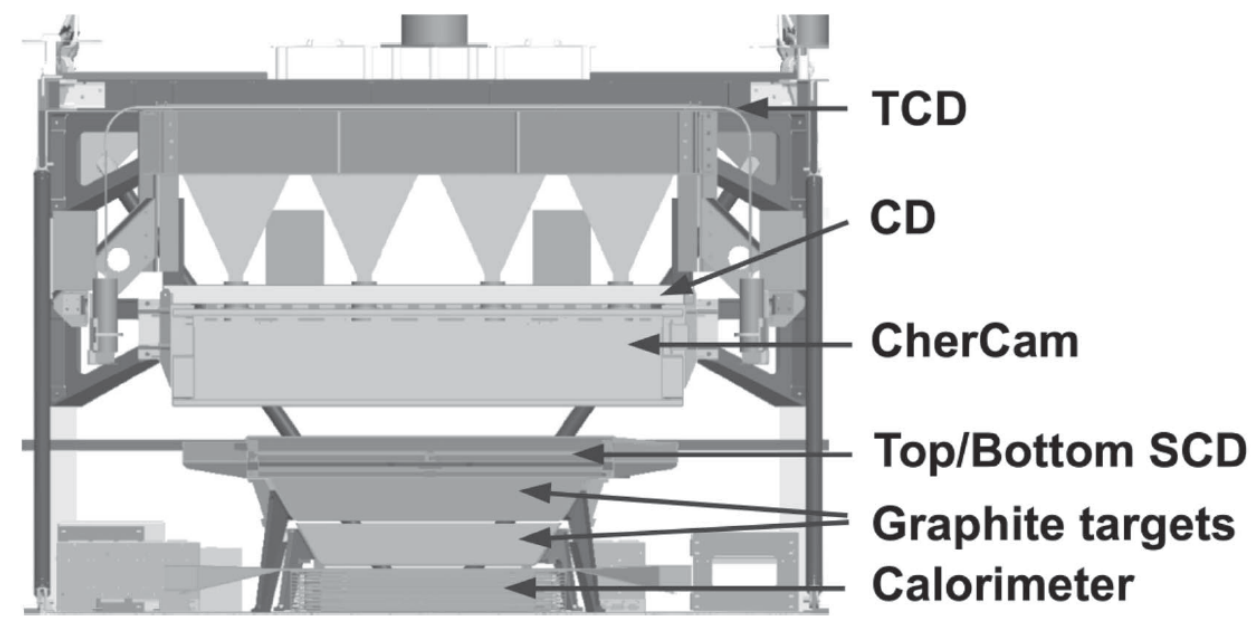

Figure 1. CREAM-III instrument; from the top: TCD, CD, CherCam, Top/Bottom SCD, graphite target blocks, and calorimeter.

The CREAM-III payload was launch from Williams Field just outside McMurdo station in Antarctica on Dec. 19, 2007. The payload circumnavigated Antarctica three times and landed on Jan. 17, 2008 for a flight time of approximately 29 day. For the duration of the flight, float altitude was between $37-40 \mathrm{~km}$ and an average atmospheric overburden of $3.9 \pm 0.4 \mathrm{~g} \mathrm{~cm}^{-2}$. Operation of the payload was conducted at the Science Operation Center at the University of Maryland. The calorimeter HV and trigger thresholds did not need any in-flight adjustments, as opposed to the first two flights where these had to be optimized. The live-time fraction of triggered science data was about $99 \%$ and $1.3 \times 10^{6}$ events were collected. The live time fractions of CREAM-I, and II were $\sim 56 \%$ and $\sim 75 \%$, respectively. The trigger energy threshold was lowered from $60 \mathrm{MeV}$, used in the first two flights, to $15 \mathrm{MeV}$ for CREAM-III, allowing for a significant increase in events around $1 \mathrm{TeV}$ [5].

\section{Data Analysis}

\subsection{Event Selection}

In flight, readout of the instrument is triggered whenever an incident cosmic ray deposits energy greater than $15 \mathrm{MeV}$ in at least one fiber ribbon in at least six geometrically consecutive layers of the CAL. Triggers from the CAL are fully efficient for interacting cosmic rays above $1 \mathrm{TeV}$. The first level of event selection is by hardware based on the CAL response and events are stored in on-board storage devices at a rate $\sim 1 \mathrm{~Hz}$. Additional levels of event selection are done using off-line software and detailed in the following sub-sections.

Work that remains to be to add includes energy deconvolution of the CAL and corresponding systematic uncertainties including variations in charge identification, and trigger efficiency. Corrections to interactions of cosmic rays up to the top of the atmosphere (TOA) and corrections to interactions of cosmic rays up to the top of the instrument (TOI) were applied to this analysis assuming the same atmostphere and instrumentation above the SCD as from the CREAM-II flight. A detailed study of the TOI corrections are needed for CREAM-III since there is a CherCam for this flight. In order to follow a conservative approach for this preliminary analysis and to compare 
CREAM-III results with previous measurements, event selection cuts presented in this work follow those described in the previous CREAM-II relative abundance studies [6].

\subsection{Energy Measurement}

The energy deposit in the calorimeter is apporximately $0.015 \%$ of incident cosmic-ray energy for protons. The CAL response was studied in beam tests using a proton beam in the energy range $50-350 \mathrm{GeV}$. Simulation indicates that the CAL response is nearly linear for nuclei above this energy. The energy resolutions for various nuclei, $5 \leq Z \leq 26$, is reported elsewhere [6], but on average is approximately $50 \%$. A calibration constant for each ribbon, determined from beam tests to be $\sim 0.08 \mathrm{MeV} / \mathrm{ADC}[1]$, was used to convert CAL signals to units of energy.

\subsection{Charge Measurement, Calculation, and Correction}

Charge is determined using both layers of the SCD and all signals were calibrated for differences in noise level, temperature and gain. Due to backscattering of cosmic rays in the carbon target, there are many pixels with signal above threshold in the SCD. In order to identify the hit position, and therefore the charge of the incident cosmic-ray, trajectories are determined by the shower axis in the calorimeter. Tracks in the CAL were constructed using a least-squares fit that requires at least 6 consecutive layers. There is a linear fit to the 10 layers in the $\mathrm{XZ}$ plane and 10 layers in the $\mathrm{YZ}$ plane. Only events that have a track reconstruction with a reduced $\chi^{2}<10$ were analyzed, as shown in Figure 2.
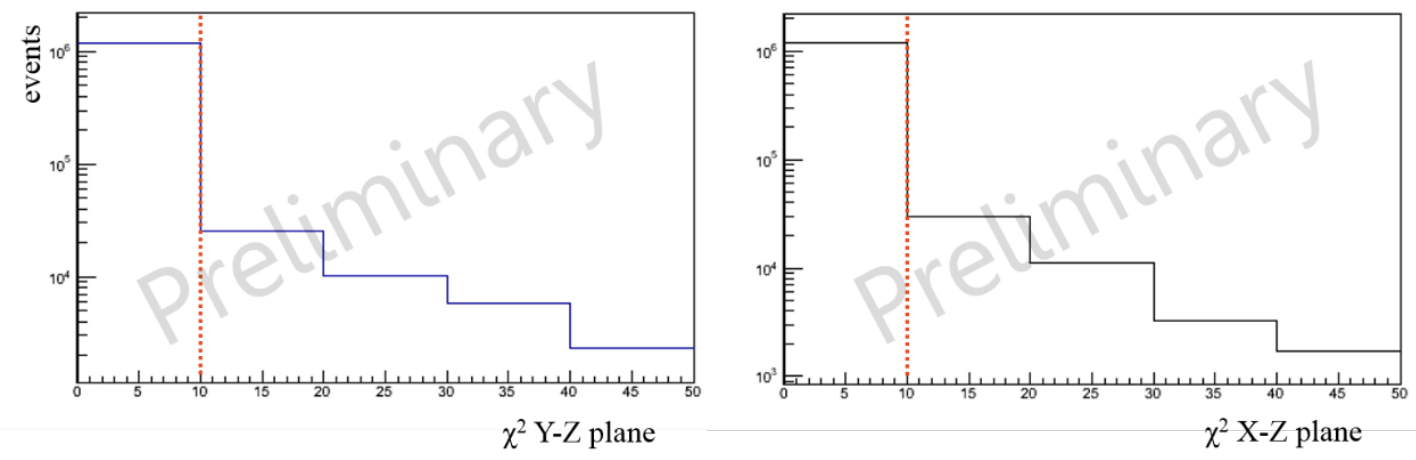

Figure 2. Distribution of $\chi^{2}$ in Y-Z and X-Z planes in the CAL. Events with $\chi^{2}$ less than 10 are kept for analysis.

The position of the track in the SCD was extrapolated and the largest signal within a radial distance of $3 \mathrm{~cm}$ from the track was chosen as the charge. Since energy loss in the SCD depends on path length, a path length correction was applied. The charge of the incident cosmic ray is proportional to the square root of the signal and the final charge used, shown in Figure 3, was an average of the two SCD signals following Equation (1), where $S_{\text {top }}$ and $S_{\text {bottom }}$ are the respective signals from the SCD layers, $\cos (\theta)$ is the path length correction, and $\mathrm{s}_{0}$ is a calibration constant, inferred from the flight data, to convert the pulse height into an absolute charge scale [7]. 


$$
Z_{\text {rec }}=\sqrt{\frac{S_{\text {top }}+S_{\text {bottom }}}{2} \times \frac{\cos (\theta)}{S_{0}}}
$$

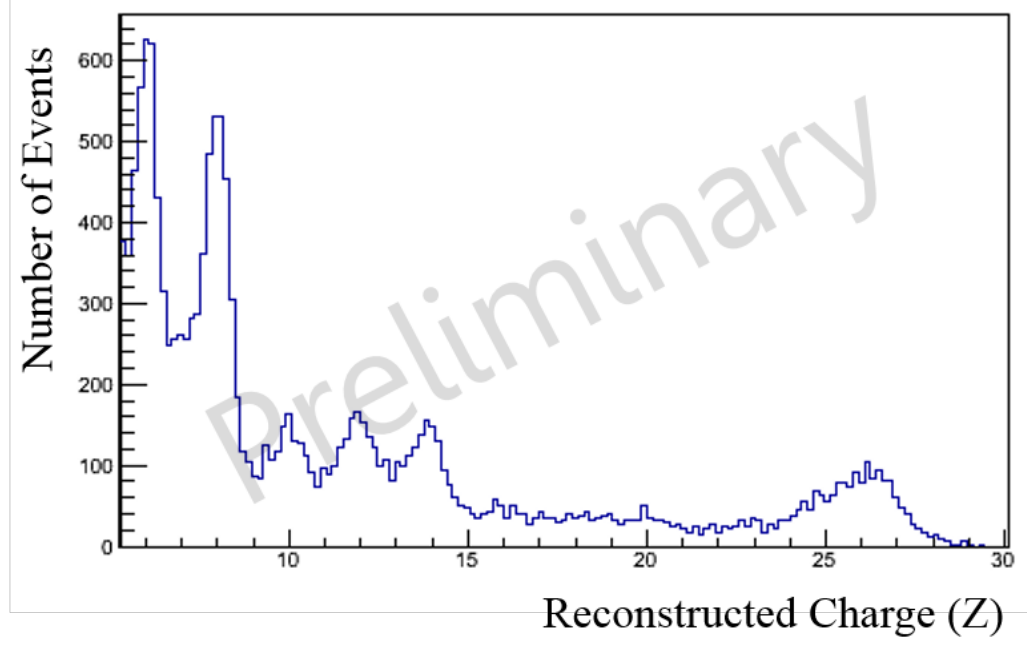

Figure 3. Distribution of reconstructed charge of events using charge consistency checks, and path length correction.

\subsection{Nuclei Selection for Relative Abundance Ratio}

A consistency cut between the charge calculated in each SCD was applied to events to cut events affected by superposition of backscattered particles, dead channels and interaction of particles between layers. The determination of consistency between the two SCD layers is based on the same calculation used for CREAM-II abundance measurements following |Ztop-Zbottom| $<\Delta \mathrm{Z}$, where $\Delta \mathrm{Z}$ starts at 0.57 for Carbon and increases linearly to 0.88 for Iron. The selection cut is illustrated in Figure 4 where the dotted line depicts the limits of consistency. 


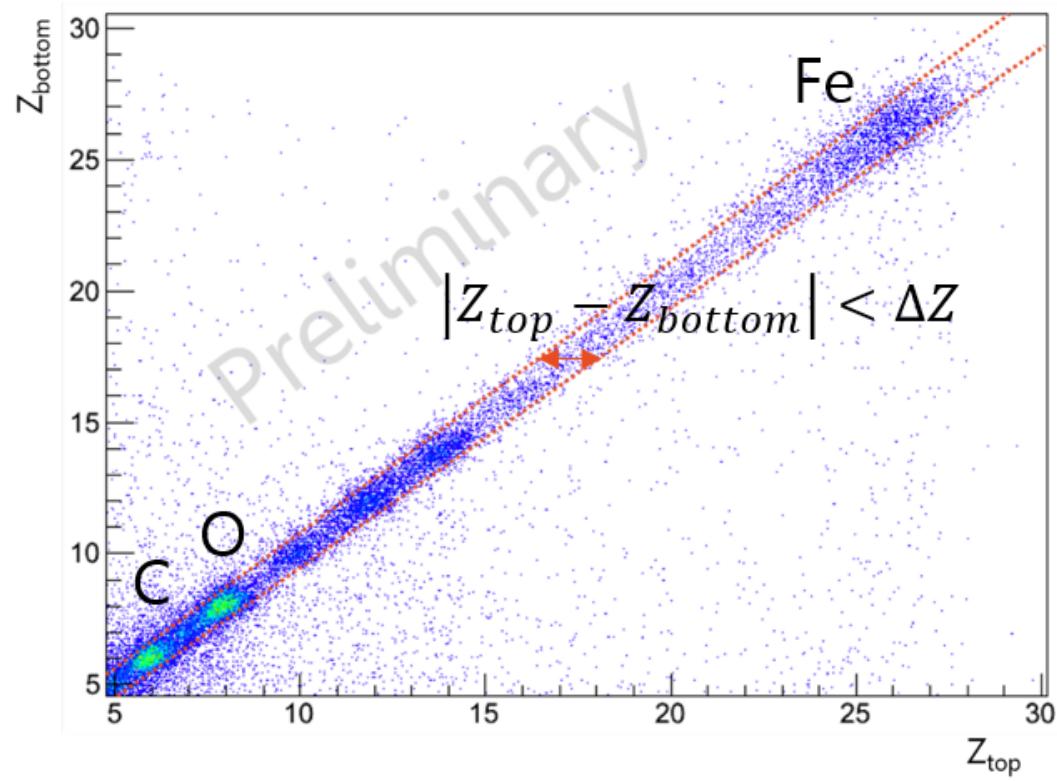

Figure 4. Consistency test of charge measurement from the dual-layer SCD with the events within red dotted lines kept for analysis.

To identify the charge peaks and widths from Figure 3, each peak was fitted with a Gaussian around the center of the peak. The results of these were then used to generate a range to select nuclei for the calculation of relative abundance. For Carbon and Oxygen a range of $2 \sigma$ around the mean was used. A range of $1 \sigma$ is used for Iron.

\section{Measured Abundance Ratios}

After applying the above tracking, consistency and selection cuts, the relative abundance ratios of elements are shown in Figure 5, with a filled square denoting the a) $\mathrm{C} / \mathrm{O}$ and b) $\mathrm{Fe} / \mathrm{O}$ ratio in each graph. Previous measurements from the CREAM-II 2010 study and CREAM-II 2009 study are shown in the figures as open triangles and open circles, respectively, as well as HEAO (open purple diamonds) and CRN (open black square). The values of the previous ratios are taken from the 2009 CREAM-II paper [6]. The statistical uncertainty in the present study is derived by error propagation of each element to a ratio, while the measured number of events is used for the statistical error without any correction of TOI or TOA at the time of this work. 


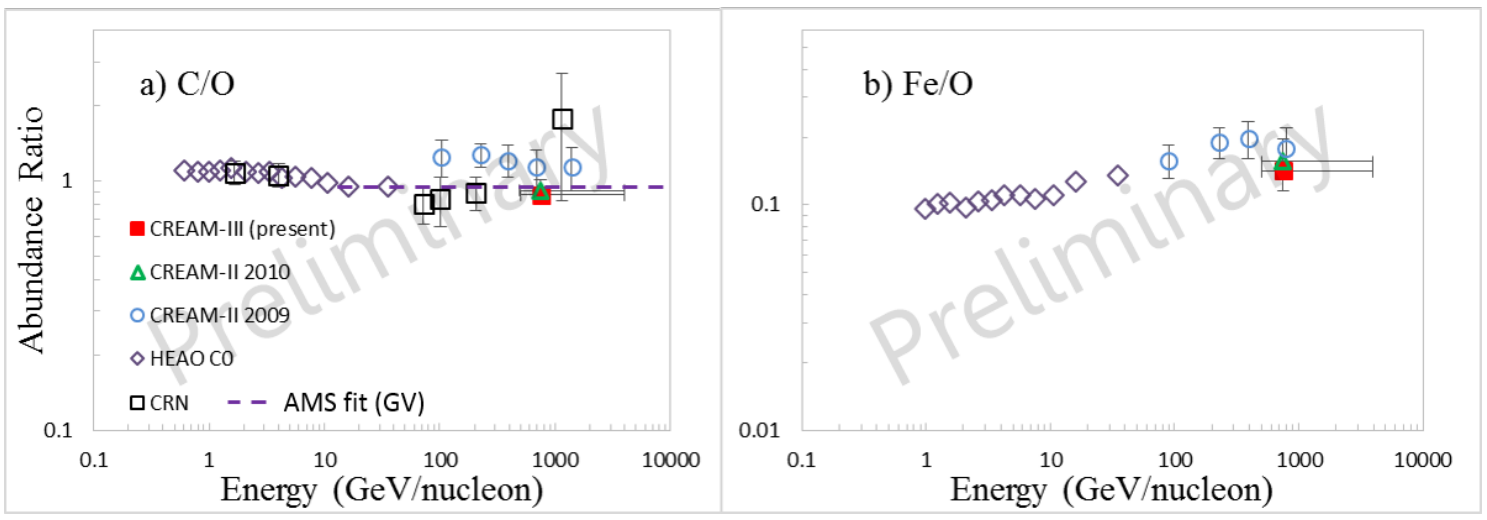

Figure 5. Relative ratios of the elements a) C/O, b) Fe/O. CREAM-III results (filled red squares) are compared with CREAM-II results (open triangles, 2010 and open circles, 2009), HEAO (open purple diamonds), CRN (open black square), and AMS fit (GV).

\section{Discussion}

The results for relative abundance ratios for $\mathrm{C}$ and $\mathrm{Fe}$ as shown in Figure 5 extend to the same energy region as the latest CREAM-II results. Ratios are corrected for secondaries produced inside the atmosphere and the instruments using the same correction factors as CREAM-II (C/O $12 \%$ reduction and $\mathrm{Fe} / \mathrm{O} 32 \%$ increase). CREAM-II had less material above the SCD so a full calculation of the TOA correction is needed for CREAM-III. The ratios of the present work are consistent with those from AMS, CRN, HEAO, and CREAM-II. The statistical uncertainties in the present study are much less than those of CREAM-II since the previous work includes a twostep tracking algorithm whereas the present work only has one that allows more events. In addition to this the live time for CREAM-III was 1.3 times more that CREAM-II flight.

\section{Conclusion}

We determine the relative elemental abundances of high-energy cosmic rays using CREAMIII flight data. Particle energy was measured using a sampling calorimeter made of alternating layers of tungsten and scintillating fiber ribbons along with a densified carbon target. Particle charge is measured and precisely identified with two layers of a SCD, previously used in the CREAM-II flight. The measured relative abundances of cosmic rays of Carbon and Iron to Oxygen in the energy range 500-3980 GeV/nucleon were found to be in good agreement with earlier measurements.

The work reported in this paper was supported in the U.S. by NASA grants NNX08AC11G, NNX08AC15G, NNX08AC16G and their predecessor grants in Korea by the Creative Research Initiatives (RCMST) of MEST/NRF and in Italy by INFN. The authors acknowledge NASA/WFF for provision and operation of flight support systems; Art Ruitberg, Suong Le, and Curtis Dunsmore of NASA/GSFC, and Carlos Urdiales of Southwest Research Institute for assistance with HV design and potting; CERN for provision of excellent accelerator beams; the Fermi National Accelerator Lab Thin Films Group for high-quality polishing and aluminization of optical elements; and Columbia Scientific Ballooning Facility, National Science Foundation's Office of Polar Programs, and Raytheon Polar Services Company for outstanding support of launch, flight and recovery operations in Antarctica. 


\section{References}

[1] Lee M.H., et al., Performance of the CREAM-III Calorimeter, ITNS, 56, 1396 (2009).

[2] Bourrion O., et al., Design and construction of a Cherenkov imager for charge measurement of nuclear cosmic rays, JInst, 6, 6004 (2011).

[3] Coutu S., et al., Design and performance in the first flight of the transition ration detector and charge detector of the CREAM balloon instrument, NIMPA, 572, 485 (2007).

[4] Nam S., et al., Performance of a dual layer silicon charge detector during CREAM balloon flight, ITNS, 54, 1743 (2007).

[5] Yoon Y.S., et al., Proton and Helium Spectra from the CREAM-III Flight, ApJ, 839, 5 (2017).

[6] Ahn H.S., et al., Measurement of the Relative Abundances of high-energy cosmic-ray nuclei in the TeV/nucleon region, ApJ, 715, 1400-1407 (2010).

[7] Ahn H.S., et al., Energy Spectra of Cosmic-Ray Nuclei at High Energies, ApJ, 707, 593-603 (2009). 